\title{
Rapamycin Combined with Immature Dendritic Cells Attenuates Obliterative Bronchiolitis in Trachea Allograft Rats by Regulating the Balance of Regulatory and Effector T Cells
}

\author{
Ming Dong ${ }^{a}$ Xin Wang ${ }^{b}$ Jie Liu ${ }^{d}$ Yu-Xia Zhao ${ }^{c}$ Xing-Long Chen ${ }^{c}$ Ke-Qiu Lic \\ Guang $\mathrm{Li}^{\mathrm{c}}$ \\ ${ }^{a}$ Department of Lung Cancer Surgery, Tianjin Medical University General Hospital, ${ }^{b}$ Department of Pediatric \\ Surgery, Tianjin Children's Hospital, and 'Basic Medical College, Tianjin Medical University, Tianjin, and \\ dSecond Department of Surgery, Cangzhou Hospital of Integrated Traditional Chinese and Western \\ Medicine of Hebei Province, Cangzhou Clinical College of Integrated Traditional Chinese and Western \\ Medicine of Hebei Medicine University, Cangzhou City, PR China
}

\section{Key Words}

Obliterative bronchiolitis - Trachea transplantation . Immature dendritic cells · Rapamycin · Regulatory T cells . Effector T cells

\begin{abstract}
Background: Obliterative bronchiolitis (OB) ranks as the major obstacle for long-term survival of lung transplantation patients. Rapamycin (Rapa) has recently been confirmed as an immunosuppressant for antirejection due to its suppressive role in T cell activation. Here, we explore the effect of Rapa combined with immature dendritic cells (imDCs) on OB in trachea allograft rats. Methods: The effect of bone marrow-derived imDCs or Rapa-imDCs on lymphocyte cells and $C D 4^{+} T$ cells were evaluated by methyl thiazolyl tetrazolium and flow cytometry. Tracheal transplantation was performed from Lewis rats to Wistar recipients. Recipient rats received Rapa+imDCs for 10 consecutive days after implantation. Allograft rejection was assessed by micro-CT image, hematoxylin/eosinHE staining and flow cytometry. The underlying mechanism was also investigated.
\end{abstract}

Results: Rapa-imDCs inhibited lymphocyte and CD4 ${ }^{+} \mathrm{T}$ cell growth. Furthermore, Rapa-imDC treatment induced T cell hyporesponsiveness by attenuating $T$ cell differentiation into IFN-p-producing $\mathrm{T}$ cells (Th1), but increased $\mathrm{CD}^{+} \mathrm{CD} 25^{+} \mathrm{Foxp}^{+} \mathrm{T}$ cell (Treg) contents. Importantly, RapaimDC administration ameliorated airway obliteration symptoms and $\mathrm{CD}^{+}$and $\mathrm{CD} 8^{+} \mathrm{T}$ cell infiltration. Furthermore, the proinflammatory factor levels of IL-6, TNF- $\alpha$, IFN- $\gamma$ and IL-17 were decreased, concomitant with the upregulation of immunosuppressive cytokines IL-10 and TGF- $\beta 1$. Further analysis confirmed that Rapa-imDC treatment attenuated the amounts of infiltrated IL- $17^{+} \mathrm{CD} 4^{+} \mathrm{T}$ cells (Th17 cells) and Th1 cells, but increased Treg contents in the spleens of recipients. Conclusions: This research may corroborate a protective role of Rapa-imDCs in OB by regulating the balance between effector $T$ cells and Tregs, suggesting a potential applicable strategy to treat $\mathrm{OB}$ after lung transplantation.

(c) 2015 S. Karger AG, Basel

M.D., X.W. and J.L. contributed equally to this work and are considered co-first authors.

\section{KARGER 125}

(c) 2015 S. Karger AG, Base

$1018-2438 / 15 / 1673-0177 \$ 39.50 / 0$

E-Mail karger@karger.com

www.karger.com/iaa
Correspondence to: Dr. Guang Li

Tianjin Medical University

No. 22 Qi Xiang Tai Road

Tianjin 300070 (PR China)

E-Mail guanglitj@163.com 


\section{Introduction}

Lung transplantation ranks as an effective strategy for the treatment of various end-stage pulmonary diseases, such as chronic obstructive pulmonary disease [1-3]. However, chronic graft dysfunction in lung transplantation is characterized by obliterative bronchiolitis (OB) and bronchiolitis obliterans syndrome, which are the leading causes of morbidity and mortality for patients with lung transplantation $[4,5]$. Though the successful application of many immunosuppressive drugs has been developed in preventing acute allograft rejection, chronic obliteration of bronchioles is still the critical cause of long-term allograft survival.

Recently, one promising way to overcome allograft rejection is the induction and maintenance of immune tolerance by cell-based therapies, such as dendritic cells (DCs) and T regulatory cells (Tregs) $[6,7]$. It is widely accepted that DCs act as the professional antigen-presenting cells to initiate immunity response by activation of native $\mathrm{T}$ cell response [8]. Furthermore, their important roles in immune tolerance have been recently recognized by deleting $\mathrm{T}$ cells or inducing Tregs $[9,10]$. DCs are known to exert various functions within the immune system depending on their maturational state. Accumulating evidence has demonstrated that the immature DCs (imDCs) of either donor or host origin are inherently tolerogenic and possess a critical function in enhancing allograft survival by suppressing $\mathrm{T}$ cell responsiveness and inducing the differentiation of Tregs, indicating an important strategy for graft survival by adoptive transfer of imDCs $[10,11]$.

$\mathrm{T}$ cells have proven to be critical for the immune response during the process of allograft rejection. A large body of research has confirmed that effector $\mathrm{T}$ cells are known to play an important role in inflammation and autoimmune diseases involving Th1 cells and Th17 cells $[12,13]$. Blocking T cell-mediated immune reactivity can prolong the survival time of grafts. As a subset of $\mathrm{CD} 4^{+} \mathrm{T}$ cells, Tregs (also known as $\mathrm{CD} 4^{+} \mathrm{CD} 25^{+}$Foxp $3^{+}$Tregs) can protect the host from autoimmunity by suppressing the proliferation and/or function of various immune cells, including natural killer cells, $\mathrm{CD} 8^{+} \mathrm{T}$ cells and $\mathrm{CD} 4^{+}$ Th1 cells. Accumulating research has confirmed a potential application of Tregs for translation rejection by promoting postoperative immunosuppression reactivity $[14$, 15].

Rapamycin (Rapa; also known as sirolimus) is a macrolide fermentation product of Streptomyces hygroscopicus and was initially explored as an antifungal and anti- tumor agent $[16,17]$. Recently, Rapa has drawn growing appreciation due to its important roles in immunosuppression $[18,19]$. Rapa is also ranked as an inhibitor for mammalian target of rapamycin (mTOR), which exerts crucial roles in adaptive immunity by keeping a proper balance between $\mathrm{T}$ cell quiescence and activation [20, 21]. It has been demonstrated that mTOR activation enhances the differentiation of native $\mathrm{T}$ cells into distinct effector T cell subsets, indicating a potential role of Rapa in autoimmunity [21]. Furthermore, Rapa treatment also slows cardiac transplantation-induced vasculopathy and promotes the survival of graft, implying a potential implication of Rapa for allograft immune response [18].

Based on the important roles of imDCs and Rapa in immune reactivity, we investigated the combined application of imDCs and Rapa in rat trachea transplantation. Additionally, the underlying mechanism was also explored.

\section{Materials and Methods}

Reagents and Antibodies

Rapa was from Sigma Chemical Company (St. Louis, Mo., USA). Anti-rat CD11b, I-Ab, CD86, CD3, CD28 and CD4-FITC were purchased from BD Pharmingen (San Diego, Calif., USA). Anti-rat mAbs for CD25, IL-17 and Foxp3 were obtained from eBioscience (San Diego, Calif., USA). Antibodies against IFN- $\gamma$ FITC were from BD Biosciences (Rockville, Md., USA). The ELISA kits for IL-6, IL-10 and IFN- $\gamma$ were purchased from BD Pharmingen. IL-17 and TNF- $\alpha$ ELISA kits were from eBioscience. TGF- $\beta 1$ ELISA kits were from R\&D Systems (Minneapolis, Minn., USA).

\section{Animals}

Fifty adult male specific pathogen-free Lewis and Wistar rats (8-10 weeks old, 200-250 g) were obtained from the Beijing Vital River Experimental Animal and Technology Co., Ltd. (Beijing, China). All rats were maintained in the specific pathogenfree animal care facilities in compliance with the guidelines of the Institutional Animal Care and Use Committee of Tianjin Medical University General Hospital. All the animal experiments were conducted according to a protocol approved by the Institutional Animal Care and Use Committee of Tianjin Medical University General Hospital. All efforts were made to minimize suffering.

\section{Isolation and Culture of Bone Marrow-Derived imDCs}

The bone marrow-derived imDCs were generated as previously described [22]. Briefly, bone marrow cells were isolated from the femur and tibia of rats in a sterile environment. Following treatment with hypotonic buffered tris-ammonium chloride $(0.83 \%$, $\mathrm{pH}$ 7.21), cells were cultured in complete RPMI 1640 medium (Corning, Manassas, Va., USA) in the presence of recombinant 
murine GM-CSF (20 ng/ml; Schering-Plough Research Institute, Kenilworth, N.J., USA) and $10 \mathrm{ng} / \mathrm{ml}$ of recombinant rat IL-4 (R\&D Systems, Minneapolis, Minn., USA) at $37^{\circ} \mathrm{C}, 5 \% \mathrm{CO}_{2}$. Then, the nonadherent cells were removed on day 2. Cells were further incubated every second day with the medium containing fresh GM-CSF and IL-4. Rapa-imDCs were prepared as above, but in the presence of Rapa (100 nM).

\section{Lymphocyte Proliferation and Apoptosis Assay}

Spleen cells were isolated from Wistar rats. Following lysis into single-cell suspensions, about $100 \mu \mathrm{l}$ of mononuclear cells $\left(5 \times 10^{6} /\right.$ well) were seeded onto 96-well plates and then cocultured with Rapa-imDCs or imDCs pretreated with mitomycin $\mathrm{C}$ at $37^{\circ} \mathrm{C}$. After incubation for $24 \mathrm{~h}$, the culture medium was replaced with fresh medium containing $500 \mu \mathrm{g} / \mathrm{ml}$ of methyl thiazolyl tetrazolium (MTT; Sigma) for an additional $5 \mathrm{~h}$ at $37^{\circ} \mathrm{C}$. The absorbance was measured at $490 \mathrm{~nm}$ on a microplate reader (Bio-Rad, Hercules, Calif., USA) to analyze cell proliferation. Cells were resuspended in medium containing $5 \mu \mathrm{l}$ of FITC-conjugated annexin $\mathrm{V}$, followed by further incubation with $5 \mu \mathrm{l}$ of PI. Cell apoptosis was assessed by flow cytometry with a FACSCalibur using the CellQuest software (BDIS), and all results were shown as a percentage of total cells counted.

\section{Splenic $\mathrm{CD}^{+} \mathrm{T}$ Cell Isolation}

Spleens from Wistar rats were obtained as described above. Then, the obtained spleens were teased into single-cell suspensions and filtered through a $70-\mu \mathrm{m}$ cell strainer (BD Falcon, USA). Following removal of the red blood cells by RBC lysing buffer (R\&D Systems), the native $\mathrm{CD} 4^{+} \mathrm{T}$ cells $\left(\mathrm{CD} 4^{+} \mathrm{CD} 25^{-}\right)$were negatively separated using the MACS CD4 ${ }^{+} \mathrm{T}$ cell Isolation Kit (Miltenyi Biotech) according to the manufacturer's instructions.

\section{In vitro Stimulation and Analysis of $\mathrm{CD} 4^{+} \mathrm{T}$ Cells}

The obtained $\mathrm{CD}^{+} \mathrm{T}$ cells were incubated with RPMI containing $10 \%$ FCS and stimulated with precoated anti-CD3 $(5 \mu \mathrm{g} / \mathrm{ml})$ and anti-CD28 $(2 \mu \mathrm{g} / \mathrm{ml})$ (both from Sanquin Research, Amsterdam, Netherlands). Then, cells were cocultured with imDCs or Rapa-imDCs for 5 days. The expression of Foxp 3 was determined by intracellular staining with the Foxp3 staining kit (BioLegend) according to the manufacturer's instructions. The percentages of IFN- $\gamma^{+}$-producing cells were evaluated by intracellular staining using anti-IFN- $\gamma$-FITC.

\section{T Cell Proliferation Assay in vitro}

To detect $\mathrm{T}$ cell proliferation induced by imDCs, the isolated $\mathrm{CD}^{+} \mathrm{T}$ cells were labeled with $2 \mu \mathrm{M}$ CFSE and stimulated with anti-CD3- and anti-CD28-coated Dynal beads. Following coculture with imDCs pretreated with Rapa for 5 days, the T cell proliferative response was determined by the CFSE dilution assay and analyzed by flow cytometry.

\section{In vivo Animal Experimental Protocol}

For orthotopic tracheal transplantation, male Lewis and Wistar rats were introduced as donors and recipients. The procedures were performed as previously described [23]. The experiments were divided into three groups ( $n=15$ in each group): control group, imDC-treated group and Rapa-imDC-treated group. For each graft, rats were anesthetized by a single intraperitoneal injection of ketamine/xylazine (100:10 mg/kg i.p.). Six or seven rings of donor trachea were removed and implanted end-to-end into the recipient via a midline cervical incision. The combined treated recipients were injected intravenously via the lateral tail vein with 2 $\times 10^{6}$ donor (Lewis) imDCs or Rapa+imDCs after implantation for 10 consecutive days. On day 30 after transplantation, the animals were sacrificed under anesthesia (sodium pentobarbital $50 \mathrm{mg} / \mathrm{kg}$, i.p.).

\section{Luminal Obliteration Evaluation}

Following anesthesia with ketamine/xylazine, the grafts of recipients were scanned by micro-CT (Explore Locus SP; GE Healthcare Company), and then the 3-dimensional images were reconstructed as previously described [23]. The obliterated areas were eventually measured by GEHC MicroView software (GE Company).

\section{Histological Analysis}

The grafted tracheal specimens were collected from recipients and fixed in $10 \%$ buffered formalin. Following dehydration, the samples were embedded in paraffin. For histological analysis, serial 4 - $\mu$ m-thick sections were obtained from each specimen, followed by staining for routine histology (hematoxylin/eosin).

\section{Analysis of Intragraft Cells}

Tracheal grafts in Wistar rats were collected and deposited in PBS. After being minced into small pieces, the specimens were dissociated using a Medimachine (BD Biosciences). Then, the 300screen mesh of copper was used to filter the homogenate. After preparation of single-cell suspensions, cells were stained with antiCD4 and anti-CD8 antibodies (BD Pharmingen) for $1 \mathrm{~h}$. Following wash with PBS three times, cells were evaluated with flow cytometry. The number of $\mathrm{CD} 4^{+}$cells and $\mathrm{CD} 8^{+}$cells in grafts was counted using BD DIVA software.

Detection of Inflammatory Cytokine Levels in Grafts by ELISA

The graft homogenate supernatants were harvested as above descript. The relative concentrations of IL- 6 , TNF- $\alpha$, IFN- $\gamma$, IL-10, IL- 17 and TGF- $\beta 1$ in homogenate supernatants were measured by ELISA kit according to the protocol provided by the manufacturers.

\section{Flow Cytometric Analysis}

After 30 days, spleen cells were collected from the recipients. Following lysis into single-cell suspensions, cells were extracellularly stained with anti-rat CD4 FITC and anti-CD8 PerCP for $1 \mathrm{~h}$. The cells were then fixed and permeabilized with a fixation/ permeabilization kit (BD Biosciences, San Diego, Calif., USA) according to the manufacturer's instructions. Following intracellular staining with anti-rat IL-17 or anti-rat Foxp3, the cell population of $\mathrm{CD} 4^{+}$Foxp $3^{+}$Treg, IL- $17^{+} \mathrm{CD} 4^{+}$Th17 and IFN$\gamma^{+} \mathrm{CD}^{+}$in spleen cells from each group was analyzed by flow cytometry (FACSCalibur with CellQuest software; BD Pharmingen).

\section{Statistical Analysis}

All values were analyzed using SPSS 13.0 software and shown as means \pm SD. The typical image was taken from at least three similar experiments. The statistical significance of differences was performed using an independent Student's t test. $\mathrm{p}<0.05$ was considered statistically significant. 


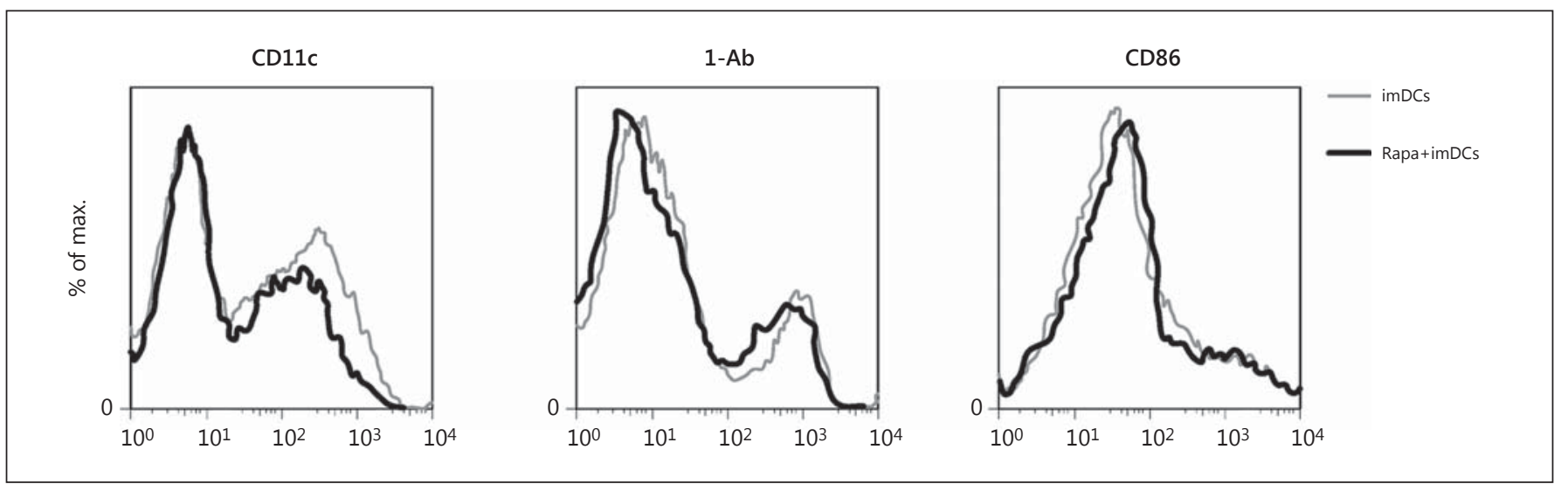

Fig. 1. Effect of Rapa-imDCs and imDCs on antigen-presenting function. The isolated bone marrow cells were cultured in complete RPMI 1640 medium. After stimulation with GM-CSF and IL-4, cells were stained with the antibodies against I-Ab, CD11c and CD86. The results were analyzed by FACS.

Fig. 2. Rapa-imDCs abrogated lymphocyte growth. Following isolation of spleen cells from Wistar rats, the mononuclear cells $\left(5 \times 10^{6} /\right.$ well $)$ were seeded onto 96 -well plates and then cocultured with RapaimDCs or imDCs pretreated with mitomycin $\mathrm{C}$ at $37^{\circ} \mathrm{C}$. a About $24 \mathrm{~h}$ later, MTT was added to analyze cell proliferation. $\mathbf{b} 5 \mu \mathrm{l}$ of FITC-conjugated annexin V was introduced to evaluate cell apoptosis by FACS. ${ }^{*} \mathrm{p}<0.05$ vs. control group. ${ }^{\#} \mathrm{p}<0.05$ vs. imDC-treated group. OD = Optical density.

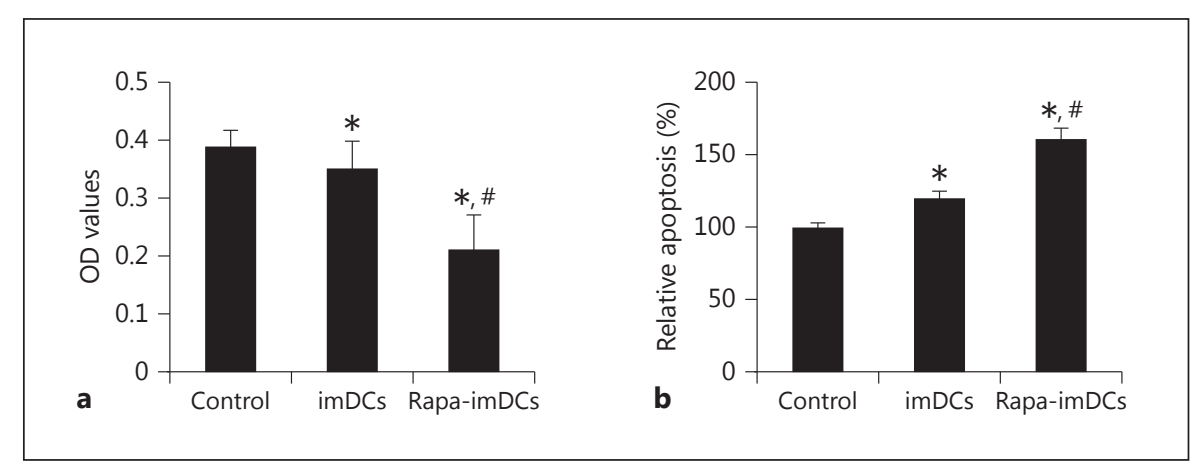

\section{Results}

Rapa-Conditioned imDCs Possessed the Potential for Antigen-Presenting Function

To explore the effect of Rapa on the surface characteristics of imDCs, the isolated bone marrow cells were stimulated with GM-CSF and IL-4. Following stimulation with Rapa, the imDCs exhibited comparable expression of MHC class II molecule I-Ab (fig. 1). Simultaneously, the expression levels of the key surface molecules of CD11c and CD86 were also observed in Rapa-imDCs and imDCs. Therefore, these results suggest that Rapa-imDCs still exert the potential for antigen-presenting function.

\section{Suppressive Capacity of Rapa-imDCs on Lymphocyte Growth}

Lymphocytes are widely accepted as a critical regulator for the survival of implants [24]. To evaluate the function of Rapa-treated imDCs on lymphocyte growth, MTT and annexin $\mathrm{V}$ were introduced to analyze cell proliferation and apoptosis. As shown in figure 2a, MTT analysis confirmed that imDC coculture attenuated cell growth of lymphocytes. When preconditioned with Rapa, the inhibitory effect on lymphocyte proliferation was further enhanced. Further apoptosis assay suggested that imDC treatment increased lymphocyte apoptosis, compared with the control group (fig. 2b). Importantly, Rapa-imDCs further upregulated cell apoptosis from 120.1 to $161.2 \%$, compared with the imDC-treated group. These data confirmed the suppressive effect of Rapa on lymphocyte growth combination with the application of imDCs, implying a potential beneficial role of Rapa-imDCs for implantation survival.

\section{Effect of Rapa-imDCs on the Development of $C D 4^{+}$}

$T$ Cells in vitro

It is known that DCs not only can initiate T cell immunity, but also induce immune tolerance by deletion of $\mathrm{T}$ cells or induction of Tregs $[8,10]$. After the isolation of na- 


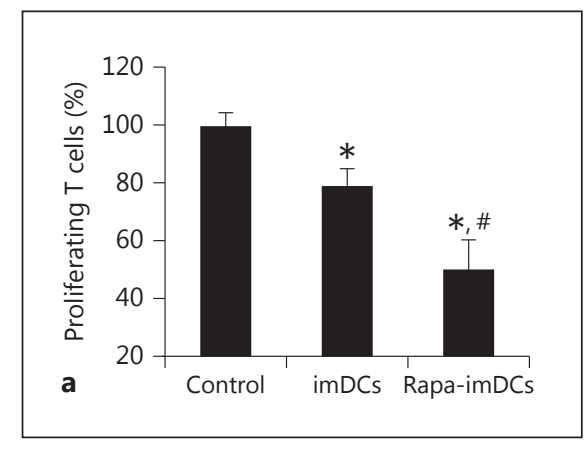

Fig. 3. Effect of Rapa-imDCs on the development of $\mathrm{CD}^{+} \mathrm{T}$ cells in vitro. Following precoating of anti-CD3 $(5 \mu \mathrm{g} / \mathrm{ml})$ and anti$\mathrm{CD} 28$, the $\mathrm{CD}^{+}{ }^{+} \mathrm{T}$ cells labeled with $2 \mu \mathrm{M}$ of CFSE from recipients were coincubated with imDCs or Rapa-imDCs. a T cell proliferative response was determined by CFSE dilution assay and analyzed by flow cytometry. b About 5 days later, the percentages of IFN- $\gamma^{+}$-producing cells were evaluated by intracellular staining using anti-IFN- $\gamma$-FITC through FACS analysis. c The Foxp $3^{+}$-producing cells were determined with a Foxp3 staining kit. ${ }^{*} \mathrm{p}<0.05$ vs. control group. ${ }^{\#} \mathrm{p}<0.05$ vs. imDC-treated group. APC = Antigen-presenting cells; $\mathrm{PE}=$ phycoerythrin.

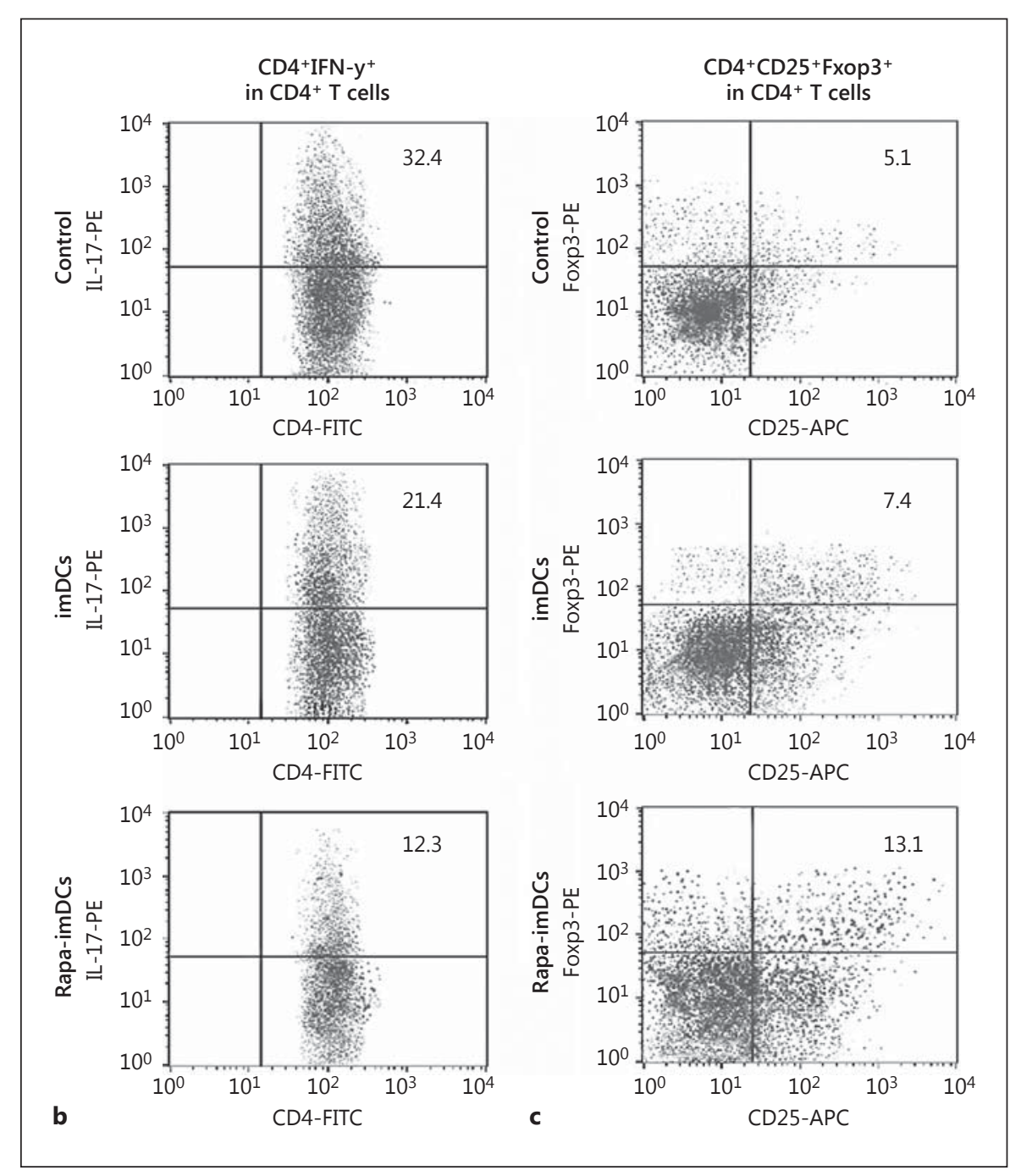

tive $\mathrm{CD} 4^{+} \mathrm{T}$ cells $\left(\mathrm{CD} 4^{+} \mathrm{CD} 25^{-}\right)$from spleens, the effect of Rapa-imDCs on $\mathrm{T}$ cell responsiveness was further evaluated. As shown in figure $3 \mathrm{a}$, the addition of imDCs significantly inhibited the proliferation of $\mathrm{CD}^{+} \mathrm{T}$ cells stimulated with anti-CD3/CD28 antibodies. Moreover, RapaimDC treatment further enhanced the inhibitor effect on $\mathrm{T}$ cell proliferation. Simultaneously, Rapa-imDC preconditioning obviously attenuated the frequency of IFN- $\gamma$ producing $\mathrm{T}$ cells (Th1) form 32.4 to $12.3 \%$, compared with the control group (fig. 3b), indicating a suppressive ability of Rapa-imDCs on Th1 differentiation. Furthermore, Rapa-imDCs showed an ability to induce Treg differentiation as evidenced by a strong increase in the frequency of Foxp $3^{+} \mathrm{CD}^{+}$-positive Tregs from 5.1 to $13.1 \%$ (fig. 3c). Together, the above results confirmed a vital function of Rapa-imDCs on $\mathrm{CD} 4^{+} \mathrm{T}$ cell hyporesponsiveness.

Rapa-imDCs Attenuate OB in Trachea Allograft
Rapa-Pretreated Donor imDCs Attenuated Allograft Rejection in Rat Trachea Transplantation

In order to assess the suppressive function of RapaimDCs in preventing allograft rejection after orthotopic tracheal transplantation, we transplanted the tracheas of Lewis rats into Wistar rats. Following the infusion of donor bone marrow-derived imDCs after transplantation, the signs of shortness of breath, mild stridor and reduced activity was mitigated to some extent at 10 days after surgery, while the recipients transplanted with a syngeneic graft showed no symptoms in the Rapa-imDC-treated group. Further analysis suggested that on day 30 after transplantation, the combination of Rapa and imDCs markedly abrogated luminal obliteration caused by submucous fibroproliferation from 58.9 to $36.1 \%$, compared with the control group (fig. 4a). We next analyzed the 

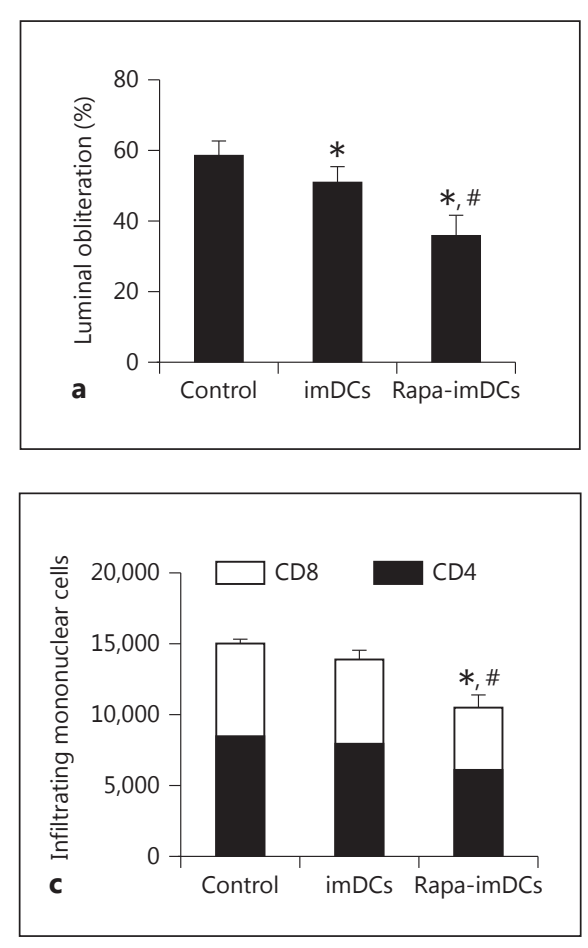

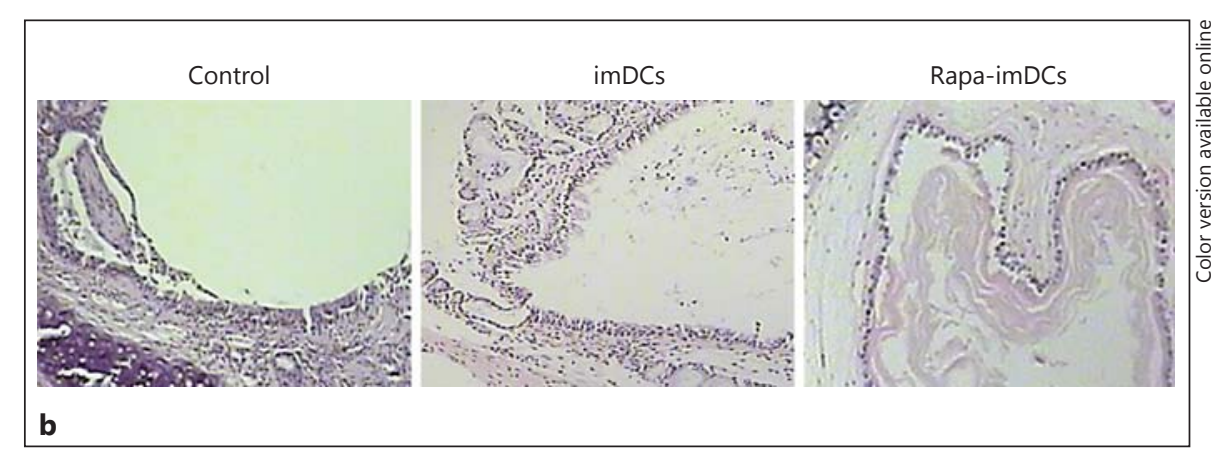

Fig. 4. Effect of Rapa-imDCs on allograft rejection in rat trachea transplantation. Following the collection of grafts from recipients treated with imDCs or Rapa-imDCs, the microCT image of orthotopic tracheal grafts was performed on postoperative day 30 , followed by the reconstruction of 3-dimensional images. a The obliteration areas were measured by GEHC MicroView software. b The serial $4-\mu \mathrm{m}$-thick sections of grafts were prepared and then stained by hematoxylin/eosin to observe the histological changes. c After preparation of single-cell suspensions, mononuclear infiltration cells were determined by staining with anti-CD4 and anti-CD8 antibodies. ${ }^{*} \mathrm{p}<0.05$ vs. control group. ${ }^{*} \mathrm{p}<0.05$ vs. imDC-treated group. histological changes of the graft by hematoxylin/eosin staining. The results indicated much inflammatory cell infiltration and many epithelial ulcerations, as well as epithelial cells appearing flattened without ciliation in grafts, which were obviously abated in the Rapa-imDC group (fig. 4b). Further FACS analysis conferred that the mononuclear infiltration of $\mathrm{CD}^{+}$and $\mathrm{CD} 8^{+}$cells in graft were dramatically reduced in the Rapa-imDC group, compared with the control group (fig. 4c). Taken together, these results show that recipients who received RapaimDCs could ameliorate clinical and histological trachea allograft rejection.

\section{Effect of Rapa-imDCs on Cytokine Production of Effector T Cells in the Graft}

To further investigate the effect of Rapa-imDCs on inflammatory cytokine levels, we collected grafts from various groups. Following lysis, the concentrations of IL-6, TNF- $\alpha$, IFN- $\gamma$, IL-10, IL-17 and TGF- $\beta 1$ in homogenate supernatants were analyzed by ELISA assay. As shown in figure $5 \mathrm{a}-\mathrm{d}$, the low amounts of proinflammatory factors were determined in imDCs and Rapa-imDCs, including IL-6, TNF- $\alpha$, IFN- $\gamma$ and IL-17. The lower levels of the above inflammatory cytokines were observed in RapaimDCs, compared with the imDC groups. Importantly, the immunosuppressive cytokines of IL-10 and TGF- $\beta 1$
To further illustrate the mechanism responsible for the protective effect of Rapa-imDCs on trachea transplantation, we analyzed the cell types of infiltrated $\mathrm{T}$ cells in the spleens of recipients. As expected, Foxp $3^{+} \mathrm{CD} 4^{+} \mathrm{T}$ cells (Tregs) in the spleens were increased in recipients treated with imDCs, compared with control groups (fig. 6a). Higher amounts of Tregs were observed in the Rapa-imDC-treated group in contrast to the imDC-treated group. Furthermore, the numbers of IL- $17^{+} \mathrm{CD} 4^{+} \mathrm{T}$ cells (Th17 cells; fig. $6 \mathrm{~b}$ ) and IFN $-\gamma^{+} \mathrm{CD} 4^{+} \mathrm{T}$ cells (Th1 cells; fig. $6 \mathrm{c}$ ) in the spleens of recipients were obviously reduced when compared with the control group. These data suggest that the Rapa-imDCs might protect trachea transplantation rejection by regulating the balance of regulatory and effector $\mathrm{T}$ cells.

\section{Discussion}

The success of lung transplantation requires the continuous administration of immunosuppressive agents to prevent and treat graft rejection. However, the severe side effects of the current immunosuppressive drugs limits their application for the treatment of graft rejection. Recently, cell-mediated therapies have become more interesting with regard to the process of allograft rejection as 


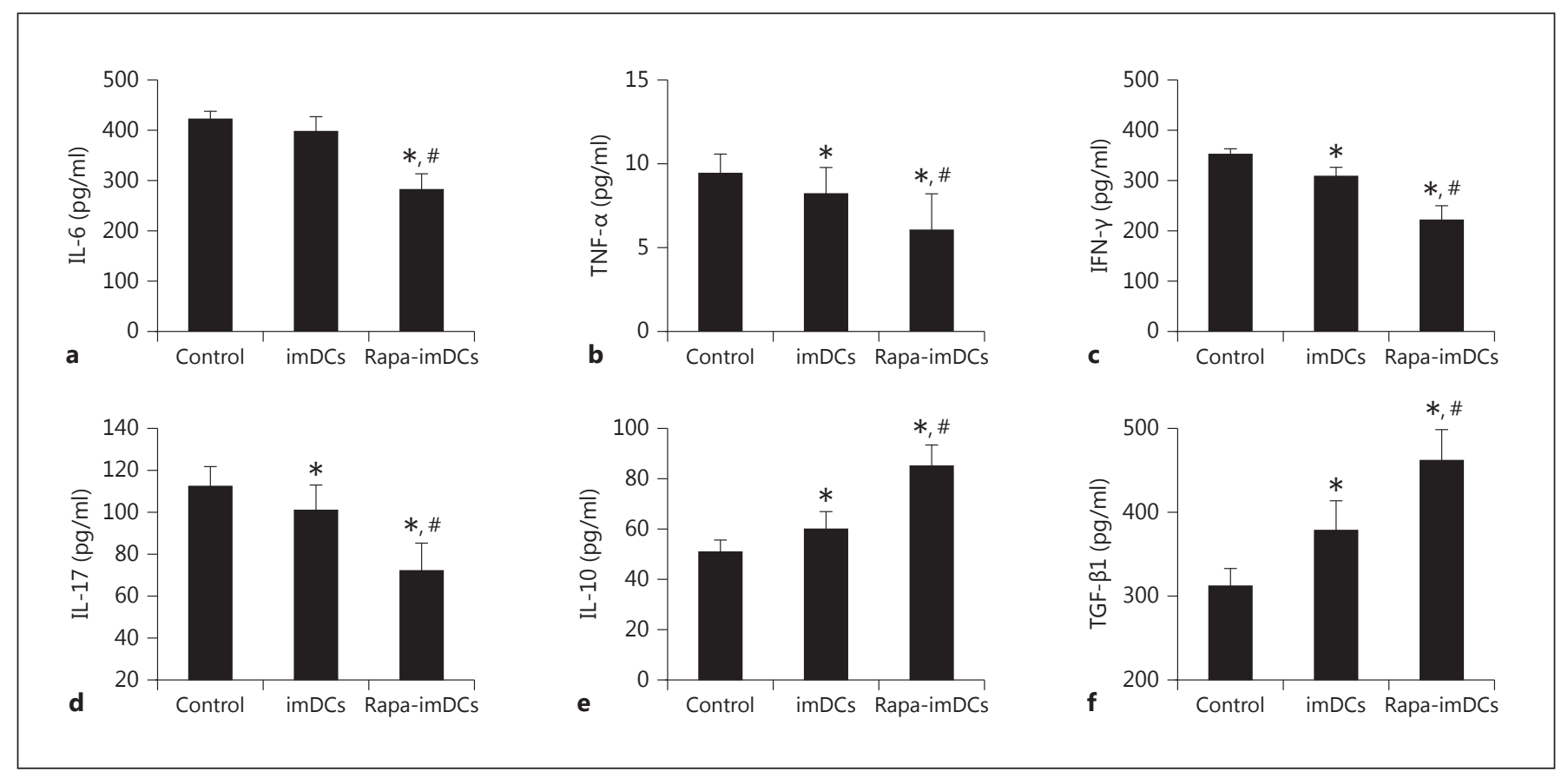

Fig. 5. Rapa-imDCs altered the expression profiles of intragraft cytokines. After the preparation of graft homogenate supernatants, the relative concentrations of IL-6 (a), TNF- $\alpha$ (b), IFN- $\gamma$ (c), IL-10 (d), IL-17 (e) and TGF- $\beta 1$ (f) in intragrafts were measured by an ELISA kit. ${ }^{*} \mathrm{p}<0.05$ vs. control group. ${ }^{*} \mathrm{p}<0.05$ vs. imDC-treated group.

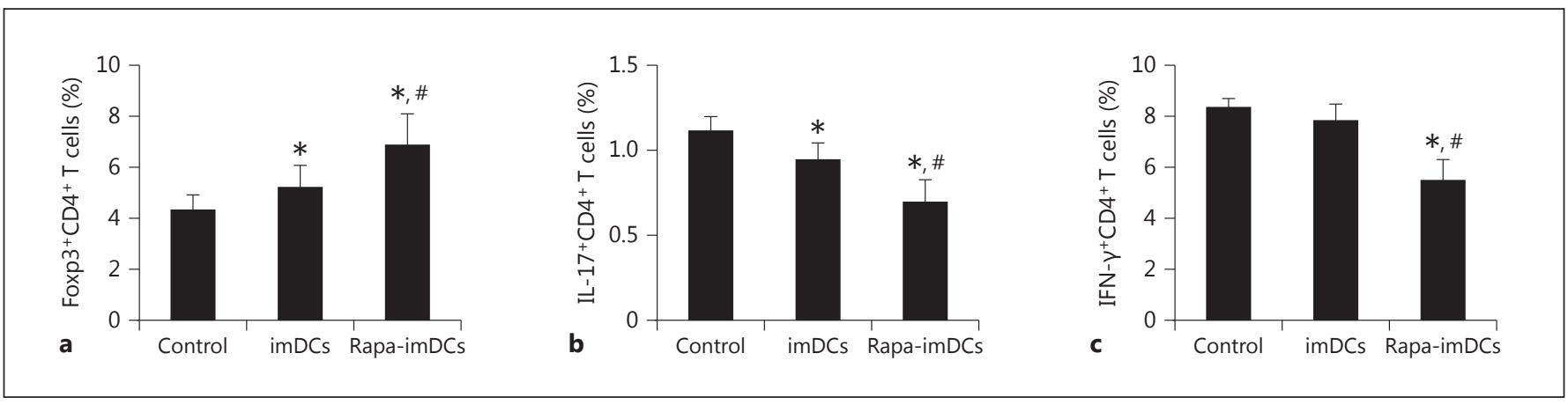

Fig. 6. Effect of Rapa-imDCs on the infiltration of effector T cells and Tregs in recipients. After 30 days, spleen cells from the various groups were collected. Following lysis into single-cell suspensions, cells were extracellularly stained with anti-rat CD4 FITC, anti-CD8. Then, the population of CD4 ${ }^{+}$Foxp3 ${ }^{+}$Tregs, $\mathrm{IL}_{-1}-7^{+} \mathrm{CD} 4^{+}$Th17 cells and IFN$\gamma^{+} \mathrm{CD} 4^{+}$Th1 cells were analyzed by flow cytometry. ${ }^{*} \mathrm{p}<0.05$ vs. control group. ${ }^{\#} \mathrm{p}<0.05$ vs. imDC-treated groups.

well as their effectiveness in the induction and maintenance of immune tolerance $[6,7]$.

DCs are commonly recognized as bone marrow-derived antigen-presenting cells and perform various functions in the immune system depending on their maturational state. Emerging evidence suggests that the imDCs usually reside in different tissues and play an important role in transplantation tolerance by inhibiting immune response $[10,11]$. After skin allograft transplantation, imDCs carrying donor antigens trigger antigen-specific immune tolerance and promote the survival of allogeneic skin grafts [25]. Rapa has recently been confirmed as an immunosuppressant for antirejection due to its suppressive role in T cell activation [14]. In this study, imDCs 
pretreated with Rapa attenuated lymphocyte proliferation and enhanced cell apoptosis, compared with the imDC-treated group, despite comparable expression of cell surface MHC class II, CD11c and CD86. Further analysis suggested a similar inhibitor effect on $\mathrm{CD}^{+}$cell proliferation stimulated with anti-CD3/CD28 antibodies. It is widely accepted that inducing the expansion of Tregs by imDCs is a common strategy for the induction of immune tolerance $[14,26]$. Here, Rapa-imDC preconditioning attenuated IFN- $\gamma$-producing T cell (Th1) differentiation. Importantly, Rapa-imDCs showed the ability to induce Treg differentiation as evidenced by a high increase in the frequency of Foxp $3^{+} \mathrm{CD} 4^{+}$-positive Tregs from native $\mathrm{CD} 4^{+} \mathrm{T}$ cells. Hence, our data suggest a vital function of Rapa-imDCs on $\mathrm{CD} 4^{+} \mathrm{T}$ cell hyporesponsiveness, indicating a potential role in graft survival.

$\mathrm{OB}$ ranks as the leading obstacle for the long-term graft survival after lung transplantation, an effective approach for the treatment of various end-stage pulmonary diseases $[4,27]$. About half of the patients with pulmonary transplantation have been demonstrated to exhibit chronic syndrome, including OB. Although whole-lung transplant models have been successfully constructed in previous research, the substantial time required for chronic obliterative lesion development and the difficulty in histologically evaluating the small airway in the lesion limit the application of lung transplant models. Therefore, to further investigate the suppressive function of Rapa-imDCs on the development of $\mathrm{OB}$, rat models with trachea transplantation were constructed. As expected, the combined administration of Rapa and imDCs markedly reduced luminal obliteration. Moreover, Rapa-imDCs also triggered decreases in inflammatory cell infiltration and ameliorated epithelial ulcerations and epithelial cells that appeared flattened without ciliation by HE staining. Additionally, mononuclear infiltration of $\mathrm{CD} 4^{+}$and $\mathrm{CD} 8^{+}$cells in grafts were dramatically reduced in the Rapa-imDC group. Therefore, these results confirmed a potential beneficial role of Rapa-imDCs in abating the development of OB.

Accumulating evidence has indicated that the severity of rejection is correlated with high inflammatory cytokines (e.g. IL-17, IFN- $\gamma$ and TNF- $\alpha$ ) and low immunosuppressive cytokines (e.g. IL-10 and TGF- $\beta 1$ ) [28-30]. In this study, Rapa-imDC treatment downregulated the amounts of proinflammatory factors in grafts, including IL-6, TNF- $\alpha$, IFN- $\gamma$ and IL-17. Simultaneously, the expression of immunosuppressive cytokines IL-10 and TGF- $\beta 1$ was dramatically increased with the combined administration of Rapa and imDCs. Although TGF- $\beta 1$ has been demonstrated to stimulate myofibroblast transdifferentiation, which contributes to matrix deposition that characterizes OB [31], a lot of research has focused on its critical function as an immunosuppressive factor in many diseases [32-34]. For heart transplantation, retinoic acid can reduce rejection in a TGF- $\beta$-dependent manner by promoting the differentiation of Tregs and inhibiting the differentiation of Th17 cells [33]. Whether TGF- $\beta$ increase can further enhance or affect the effect of Rapa-imDCs on OB will be explored in our next plan. It is recognized that the effector T cells including Th17 cells and Th1 cells are related with immune response after organ transplantation and are critical for graft survival $[6,35,36]$. Both of them can induce abundant inflammatory cytokine secretions. However, $\mathrm{CD} 4^{+} \mathrm{CD} 25^{+} \mathrm{Foxp}^{+}$Tregs possess various functions in different pathological processes, such as immune tolerance, inflammation and allograft rejection $[15,37,38]$. To further explore the underlying mechanism involved in Rapa-imDC-ameliorated OB, we investigated cell types of infiltrated $\mathrm{T}$ cells in the spleens of recipients with orthotopic tracheal transplantation. As expected, the increased amounts of Foxp $3^{+} \mathrm{CD}^{+} \mathrm{T}$ cells (Tregs) were observed in Rapa-imDCs, concomitant with the notable decrease in the numbers of IL- $17^{+} \mathrm{CD} 4^{+} \mathrm{T}$ cells (Th17 cells) and IFN$\gamma^{+} \mathrm{CD}^{+} \mathrm{T}$ cells (Th1 cells), indicating that Rapa-imDCs may exert the protective effect on trachea transplantation by inhibiting effector $\mathrm{T}$ cells and enhancing Tregs.

In conclusion, Rapa-imDCs induced lymphocyte growth inhibition and T cell hyporesponsiveness, including inhibiting $\mathrm{T}$ cell proliferation and differentiation into Th1 cells, but increasing the differentiation into Tregs. Further analysis confirmed that Rapa-imDCs attenuated airway obliteration in rat orthotopic trachea transplantation, possibly by inhibiting effector T cells and enhancing Tregs. Accordingly, this study may corroborate the protective role of Rapa-imDCs on the development of $\mathrm{OB}$ in trachea transplantation by regulating the balance of effector $\mathrm{T}$ cells and Tregs, suggesting a potential clinically applicable strategy to treat $\mathrm{OB}$ after lung transplantation.

\section{Acknowledgement}

This work was supported by the National High Technology Research and Development Program of China (863 Program: 2012AA021003).

\section{Disclosure Statement}

The authors report no proprietary or commercial interest in any product mentioned or concept discussed in this article. 


\section{References}

$>1$ Hartert M, Senbaklavaci Ö, Gohrbandt B, 15 Bascom R, Tao K, Tollenaar S, West L: ReguFischer BM, Buhl R, Vahl CF: Lung transplantation: a treatment option in end-stage lung disease. Dtsch Arztebl Int 2014;111:107-116.

$>2$ Munson JC, Christie JD, Halpern SD: The societal impact of single versus bilateral lung transplantation for chronic obstructive pulmonary disease. Am J Respir Crit Care Med 2011;184:1282-1288.

$\checkmark 3$ Abdulaziz S, Al-Shahid M, Al-Thenayan E: A 49-year-old man with acute pulmonary hypertension post lung transplantation complications of lung transplantation. Chest 2013; 144:704-707.

4 Weber DJ, Wilkes DS: The role of autoimmunity in obliterative bronchiolitis after lung transplantation. Am J Physiol Lung Cell Mol Physiol 2013;304:L307-L311.

$>5$ Paraskeva M, Bailey M, Levvey B, Griffiths A, Kotsimbos T, Williams T, Snell G, Westall G: Cytomegalovirus replication within the lung allograft is associated with bronchiolitis obliterans syndrome. Am J Transplant 2011;11: 2190-2196.

-6 Lee K, Nguyen V, Lee KM, Kang SM, Tang Q: Attenuation of donor-reactive $\mathrm{T}$ cells allows effective control of allograft rejection using regulatory $\mathrm{T}$ cell therapy. Am J Transplant 2014;14:27-38.

7 Sun X, Gong ZJ, Wang ZW, Li T, Zhang JY, Sun HC, Liu S, Huang L, Huang C, Peng ZH: IDO-competent-DCs induced by IFN- $\gamma$ attenuate acute rejection in rat liver transplantation. J Clin Immunol 2012;32:837-847.

$>8$ Lanzavecchia A, Sallusto F: Regulation of T cell immunity by dendritic cells. Cell 2001; 106:263-266.

$>9$ Saei A, Hadjati J: Tolerogenic dendritic cells: key regulators of peripheral tolerance in health and disease. Int Arch Allergy Immunol 2013;161:293-303.

10 Gao XW, Fu Y, Li WJ, Du AJ, Li X, Zhao XD: Mechanism of immune tolerance induced by donor derived immature dendritic cells in rat high-risk corneal transplantation. Int J Ophthalmol 2013;6:269-275.

$>11$ Sheng Sun D, Iwagaki H, Ozaki M, Ogino T, Kusaka S, Fujimoto Y, Murata H, Sadamori H, Matsukawa H, Tanaka N: Prolonged survival of donor-specific rat intestinal allograft by administration of bone-marrow-derived immature dendritic cells. Transpl Immunol 2005;14:17-20.

$>12$ Petermann F, Korn T: Cytokines and effector T cell subsets causing autoimmune CNS disease. FEBS Lett 2011;585:3747-3757.

13 Kuchroo VK, Korn T: Th17 cells in autoimmune inflammation and demyelination in the central nervous system. Clin Dev Immunol 2013;2013:986789.

-14 Wang X, Wang W, Xu J, Hong J, Le Q: Pretreatment of rapamycin before allogenic corneal transplant promotes graft survival through increasing CD4(+)CD25(+)Foxp3(+) regulatory t cells. Exp Clin Transplant 2013;11:56-62. latory T cells (Tregs) in neonatal tolerance: allogeneic Tregs regulate the neonatal immune system and prolong heart graft survival. J Heart Lung Transplant 2013;32:S297.

16 Anisimov VN, Zabezhinski MA, Popovich IG, Piskunova TS, Semenchenko AV, Tyndyk ML, Yurova MN, Rosenfeld SV, Blagosklonny MV: Rapamycin increases lifespan and inhibits spontaneous tumorigenesis in inbred female mice. Cell Cycle 2011;10:4230-4236.

17 Bastidas RJ, Shertz CA, Lee SC, Heitman J, Cardenas ME: Rapamycin exerts antifungal activity in vitro and in vivo against Mucor cir cinelloides via FKBP12-dependent inhibition of Tor. Eukaryot Cell 2012;11:270-281.

18 Mancini D, Pinney S, Burkhoff D, LaManca J, Itescu S, Burke E, Edwards N, Oz M, Marks AR: Use of rapamycin slows progression of cardiac transplantation vasculopathy. Circulation 2003; 108:48-53.

19 Gagliani N, Gregori S, Jofra T, Valle A, Stabilini A, Rothstein DM, Atkinson M, Roncarolo MG, Battaglia M: Rapamycin combined with Anti-CD45RB mAb and IL-10 or with G-CSF induces tolerance in a stringent mouse model of islet transplantation. PLoS One 2011;6:e28434.

$>20$ Weichhart T, Costantino G, Poglitsch M, Rosner M, Zeyda M, Stuhlmeier KM, Kolbe T, Stulnig TM, Hörl WH, Hengstschläger M: The TSC-mTOR signaling pathway regulates the innate inflammatory response. Immunity 2008;29:565-577.

21 Liu Y, Zhang DT, Liu XG: mTOR signaling in $\mathrm{T}$ cell immunity and autoimmunity. Int Rev

22 Bhatt S, Qin J, Bennett C, Qian S, Fung JJ, Hamilton TA, Lu L: All-trans retinoic acid induces arginase- 1 and inducible nitric oxide synthase-producing dendritic cells with $\mathrm{T}$ cell inhibitory function. J Immunol 2014;192: 5098-5108.

23 Shi Q, Niu Y, Cao H, Zhou X, Jiang S, Liu Z, Fan H: CD28 superagonist antibody treatment attenuated obliterative bronchiolitis in rat allo-orthotopic tracheal transplantation by preferentially expanding Foxp3-expressing regulatory T cells. Transplant Proc 2012; 44:1060-1066.

24 Motomura T, Shirabe K, Mano Y, Muto J, Toshima T, Umemoto Y, Fukuhara T, Uchiyama H, Ikegami T, Yoshizumi T: Neutrophil-lymphocyte ratio reflects hepatocellular carcinoma recurrence after liver transplantation via inflammatory microenvironment. J Hepatol 2013;58:58-64.

25 Xin $\mathrm{H}$, Yang W, Wang Q, You B, Tong Y, Peng Y: Immune tolerance of skin allograft transplantation induced by immature dendritic cells of a third party carrying donor antigens in mice. Transplant Proc 2013;45:552-557. Immunol 2015;34:50-66.
26 Choi YS, Jeong JA, Lim DS: Mesenchymal stem cell-mediated immature dendritic cells induce regulatory $\mathrm{T}$ cell-based immunosuppressive effect. Immunol Invest 2012;41:214229.

27 Barker AF, Bergeron A, Rom WN, Hertz MI: Obliterative bronchiolitis. N Engl J Med 2014; 370:1820-1828.

28 Camargo PC, Afonso JE Jr, Samano MN, Acencio MMP, Antonangelo L, Teixeira RH: Cytokine levels in pleural fluid as markers of acute rejection after lung transplantation. J Bras Pneumol 2014;40:425-428.

29 Brunet M: Cytokines as predictive biomarkers of alloreactivity. Clin Chim Acta 2012;413: 1354-1358.

30 De Serres SA, Mfarrej BG, Grafals M, Riella LV, Magee CN, Yeung MY, Dyer C, Ahmad $\mathrm{U}$, Chandraker A, Najafian N: Derivation and validation of a cytokine-based assay to screen for acute rejection in renal transplant recipients. Clin J Am Soc Nephrol 2012;7:10181025.

31 Ramirez AM, Shen Z, Ritzenthaler JD, Roman J: Myofibroblast transdifferentiation in obliterative bronchiolitis: TGF- $\beta$ signaling through Smad3-dependent and -independent pathways. Am J Transplant 2006;6:208020888.

32 Levings MK, Bacchetta R, Schulz U, Roncarolo MG: The role of IL-10 and TGF-beta in the differentiation and effector function of $\mathrm{T}$ regulatory cells. Int Arch Allergy Immunol 2002; 129:263-276.

33 Wang G, Zhong A, Wang S, Dong N, Sun Z, Xia J: Retinoic acid attenuates acute heart rejection by increasing regulatory $\mathrm{T}$ cell and repressing differentiation of Th17 cell in the presence of TGF- $\beta$. Transpl Int 2010;23:986997.

34 Donkor MK, Sarkar A, Li MO: TGF- $\beta 1$ produced by activated CD4(+) T cells antagonizes $\mathrm{T}$ cell surveillance of tumor development. Oncoimmunology 2012;1:162-171.

35 Shilling RA, Wilkes DS: Role of Th17 cells and IL-17 in lung transplant rejection. Semin Immunopathol 2011;33:129-134.

36 Chadha R, Heidt S, Jones ND, Wood KJ: Th17: contributors to allograft rejection and a barrier to the induction of transplantation tolerance? Transplantation 2011;91:939-945.

37 Dummer CD, Carpio VN, Gonçalves LFS, Manfro RC, Veronese FV: Foxp3+ regulatory $\mathrm{T}$ cells: from suppression of rejection to induction of renal allograft tolerance. Transpl Immunol 2012;26:1-10.

38 Stewart CA, Metheny H, Iida N, Smith L, Hanson M, Steinhagen F, Leighty RM, Roers A, Karp CL, Müller W: Interferon-dependent IL-10 production by Tregs limits tumor Th17 inflammation. J Clin Invest 2013;123:48594874 . 